\title{
Analytic theory for the selection of a two-dimensional needle crystal at arbitrary Péclet number
}

\author{
S. Tanveer \\ Mathematics Department, Virginia Polytechnic Institute and State University, Blacksburg, Virginia 24061
}

(Received 9 March 1989)

\begin{abstract}
An accurate analytic theory is presented for the velocity selection of a two-dimensional needle crystal for arbitrary Péclet number for small values of the surface-tension parameter. The velocity selection is caused by the effect of transcendentally small terms that are determined by analytic continuation to the complex plane and analysis of nonlinear equations. The work supports the general conclusion of previous small-Péclet-number analytical results of other investigators, although there are some discrepancies in details. It also addresses questions raised by a recent investigator on the validity of selection theory owing to assumptions made on shape corrections at large distances from the tip.
\end{abstract}

\section{INTRODUCTION}

The problem of the growth of a needle crystal in a pure undercooled liquid in the absence of any boundaries has received considerable attention in recent literature. In particular, the growth of a steadily moving interface between solid and liquid has been studied using both analytical $^{1-3}$ and numerical methods. ${ }^{4,5}$ When surface tension is neglected, exact solutions with a parabolic crystal-melt interface were found earlier by Ivanstov. ${ }^{6}$ These solutions form a degenerate set since for given undercooling and other experimental conditions, only the product of the tip radius of curvature and the steady dendrite velocity are determined in contradiction to experimental evidence $^{7,8}$ which suggests that each of these are separately determined for given undercooling far ahead of the interface. This degeneracy is not unexpected since in the absence of surface tension, there is not enough dimensional information to predict each of these physical quantities separately.

When surface tension is taken into account, there is enough dimensional information to determine each dendrite velocity and tip curvature in terms of undercooling. However, this need not imply that a solution exist in this case. Numerical evidence $e^{4,5}$ appears to suggest that such solutions do not exist if we neglect the effect of crystalline anisotropy.

Earlier, analytic study of phenomenological models 9,10 of solidification, suggested that solutions do not exist when anisotropy is neglected. The mathematical equations arising out of one of the phenomenological models ${ }^{10}$ has been rigorously studied by Kruskal and Segur. ${ }^{11}$ They prove that in the limit of zero surface tension, these model equations do not have any physically acceptable solutions when crystalline anisotropy is not taken into account even though the equations admit solutions when surface tension is exactly zero. This extraordinary situation arises due to the effect of terms beyond all orders in an asymptotic expansion for small surface tension. Kruskal and Segur extend earlier methods ${ }^{12}$ for linear equations to extract transcendentally small terms in the asymptotic expansion of the solution to the third-order nonlinear ordinary differential equation that they study and show that the leading-order transcendental correction to a regular perturbation expansion fails to satisfy the condition on smoothness of the needle crystal right at the tip. However, when a term modeling crystalline anisotropy is included in the equations, a discrete set of solutions is found to exist. However, it is not clear to us that the simple model equations studied by Kruskal and Segur should faithfully reflect the properties of the actual needle crystal, even qualitatively.

In the limit of small Péclet number, Pelce and Pomeau ${ }^{13}$ reduce the original integro-differential equation called the Nash-Glicksman equation ${ }^{14}$ to a simpler set of equations involving just one parameter. Subsequently analysis of Ben-Amar and Pomeau ${ }^{1}$ of this equation and by Barbieri and Langer ${ }^{2}$ of a simpler linearized form in the limit of small values of a certain nondimensional surface-tension parameter support the conclusions of the numerical work at arbitrary Péclet number ${ }^{4,5}$ that needle-crystal solutions do not exist in two-dimensional (2D) or axisymmetric 3D case if crystalline anisotropy is neglected. On modeling the fourfold crystalline anisotropy by a cosine term, the numerical work based on the Nash-Glicksman equation and analysis based on PelcePomeau equations suggest that a discrete set of solutions exists for any nonzero crystalline anisotropy. The analytical work of Ben Amar and Pomeau formally extends the Kruskal-Segur ${ }^{11}$ method for extracting transcendentally small terms to a nonlinear integro-differential equation. This follows earlier work of Combescot et al., ${ }^{15}$ who use the Kruskal-Segur method to Saffman-Taylor finger problem, which again involves a similar nonlinear integrodifferential equation. The work of Barbieri et al. ${ }^{2}$ is based on an approximate linear equation and is based on Fredholm alternative condition on a nonhomogeneous linear equation, where WKB approximate methods are used to find independent solutions to the homogeneous problem. This work follows the idea of Shraiman, ${ }^{16}$ who employed a similar method for the Saffman-Taylor problem. Despite the apparent deficiency of such an ap- 
proach in that the linear equations are approximate and that the WKB solutions are not quite correct in the neighborhood of turning points which must be encountered in evaluating the Fredholm condition using a steepest-descent contour in the complex plane, the scalings in the dependence of physical quantities on each other turn out to be the same as the nonlinear analysis of Ben Amar and Pomeau, the only discrepancy being in the values of constants.

However, for the axisymmetric 3D needle crystal, contradictory analytical evidence has recently been presented by $\mathrm{Xu} .{ }^{17}$ Rather than working with the Nash-Glicksman equation, he considers the original partial differential equations on both sides of the crystal-melt interface and obtains simplifications for small Péclet number using a slender body approximation. His analysis is not restricted to small surface tension. The basic approach used in his case is as follows. Given a slender axisymmetric 3D crystal, he finds an expression for the temperature in terms of an interfacial shape function. To the leading order, as Péclet number tends to zero, this expression is found to be a local function of the shape function, in contradiction with the Pelce-Pomeau simplification where the temperature at any point on the interface is expressed in terms on a global integral expression involving the shape function. Once the expression for the temperature on the needle boundary is found in terms of the shape function, $\mathrm{Xu}$ uses the Gibbs-Thompson condition of local equilibrium to reduce the problem to a second-order nonlinear ordinary differential equation which he then solves using phase plane analysis. He concludes that axisymmetric 3D needle crystals exist in the absence of crystalline anisotropy and further, even for nonzero surface tension, each dendrite velocity and tip radius is not a uniquely determined function of the undercooling. The degeneracy of the solutions is found to be the same as that for zero surface tension. Xu explains the discrepancy of his results with others by suggesting that the other researchers have implicitly assumed that the shape correction to Ivantsov parabola for nonzero surface tension tends to zero at infinity and thus have restricted the class of allowable shapes in their analysis and numerical computation. Thus the entire selection theory, at least in the axisymmetric 3D case, has been questioned. Despite some lively debate, ${ }^{18}$ this controversy is yet to be settled conclusively. We find that Xu's objections have some merit as far as the analytical evidence based on the Pelce-Pomeau equation even for the simpler 2D needlecrystal problem, since in the derivation of the PelcePomeau equation from the original Nash-Glicksman equation, it appears to be necessary to assume that the shape modifications to the Ivantsov solution tend to zero at infinity. Further, in the analytical work based on the Pelce-Pomeau equation, the integrand is linearized based on the assumption that the correction to the Ivantsov solution is small for sufficiently small surface tension. However, there does not seem to be any a priori reason to assume that for any small but fixed surface tension, the shape correction is small for the entire range of integration in the integral term and so linearization becomes questionable. As far as numerical evidence, $\mathrm{Xu}$ suggests that by truncating the infinite range of integration to a finite one and matching to the shape of a parabola at sufficiently large distances, one implicitly rules out shape corrections to the Ivantsov solutions that grow at large distances, though at a rate smaller than for a parabola. It is not clear to us if this argument has any merit or not.

What is clear from all this is that one needs to resolve the discrepancy between the work of $\mathrm{Xu}$ and other researchers. Indeed, one can make a direct check on $\mathrm{Xu}$ 's leading-order asymptotic expression for the temperature field on the 3D axisymmetric needle boundary by a careful direct asymptotics of the integral term in the Nash-Glicksman equation for small Péclet number and checking if the expression is local or global. If the PelcePomeau equation holds, then one needs to check the steps in Xu's analysis leading up to the expression for the temperature field in terms of the shape function to find possible sources of error. This is currently under study.

In the meantime, we thought it appropriate to reconsider the easier 2D needle-crystal problem, where Xu's objections have some validity as well. Instead of considering the Pelce-Pomeau simplification for small Péclet number, we thought it appropriate to consider the NashGlicksman equation for arbitrary Péclet number. When this paper was first written, we were unaware of any analytical work at arbitrary Péclet number, though the problem has been solved numerically. Since then we received a preprint of work at arbitrary Péclet number by Barbieri and Langer ${ }^{19}$ where they consider the needle crystal at arbitrary Péclet number in $2 \mathrm{D}$ as well as $3 \mathrm{D}$ using an approximate equation where the curvature term is linearized together with the integral term in the NashGlicksman equation. Aside from some quantitative errors in the value of constants that such a linearization would produce, their work does not address the objection of $\mathrm{Xu}$ as far as a priori assumption on the nature of shape correction at infinity.

While our analysis is not mathematically rigorous either, as we address some of the questions regarding linearization of the integral term in the Nash-Glicksman equation with some care. Our final conclusions suggest that the selection theory, at least in $2 \mathrm{D}$, is correct. The second-order nonlinear differential equation that arise in connection to calculating the leading-order transcendentally small correction is found to be about the same as that coming out of the Ben Amar and Pomeau ${ }^{1}$ analysis though their starting point was the simpler Pelce-Pomeau equation valid only for small Péclet number. We disagree with Ben-Amar and Pomeau on several fine points in the analysis of these nonlinear equations particularly when the crystalline anisotropy is nonzero. In particular, the predicted quantitative constant for the first branch of solution corresponding to the dendrite with the largest velocity is found to be a little different from what we predict because we believe they use an analytic expression valid only for the higher branches of solution.

\section{MATHEMATICAL FORMULATION}

In the frame of the steadily moving needle crystal, we fix the origin of the coordinate system $(x, z)=(0,0)$ at the 
tip. Let the $z$ axis be aligned in the direction of the crystal axis and the $x$ axis perpendicular to it. A point on the needle boundary is described by the parametric representation $(x(\xi), z(\xi))$, where $\xi$ is the interval $(-\infty, \infty)$ and

$$
\begin{aligned}
& z(\xi)=-\frac{1}{2} \xi^{2}, \\
& x(\xi)=\xi+x_{R}(\xi),
\end{aligned}
$$

where $x_{R}(0)=0$ in order that tip of the dendrite to be at $(0,0)$. The above parametric representation $(x(\xi), z(\xi))$ is found to be rather suitable for avoiding nonuniformity in the linearization of the Nash-Glicksman integral expression for the temperature as shall be seen shortly. The Nash-Glicksman equation for determination of the dendrite boundary can then be written as

$$
\begin{aligned}
& \Delta-d_{0}\{1+\alpha[1-\cos (4 \theta)\} \kappa \\
& =\frac{P}{\pi} \int_{-\infty}^{\infty} d \xi^{\prime}\left[1+x_{R}\left(\xi^{\prime}\right)\right] e^{P\left[z\left(\xi^{\prime}\right)-z(\xi)\right]} K_{0}(P|r|),
\end{aligned}
$$

where

$$
\begin{aligned}
r=\left(\xi-\xi^{\prime}\right)[1 & +\frac{1}{4}\left(\xi+\xi^{\prime}\right)^{2}+2 \frac{x_{R}(\xi)-x_{R}\left(\xi^{\prime}\right)}{\xi-\xi^{\prime}} \\
& \left.+\left[\frac{x_{R}(\xi)-x_{R}\left(\xi^{\prime}\right)}{\xi-\xi^{\prime}}\right]^{2}\right]^{1 / 2} .
\end{aligned}
$$

In (3), $\theta$ is the angle between the normal to the interface and the positive $z$ direction and $\kappa$ is the curvature of the interface $(x(\xi), z(\xi))$ given by

$$
\begin{aligned}
& \tan \theta=\frac{z_{\xi}}{1+x_{R \xi}}, \\
& \kappa=\frac{1+x_{R \xi}-\xi x_{R \xi \xi}}{\left(1+2 x_{R \xi}+x_{R \xi}^{2}+\xi^{2}\right)^{3 / 2}},
\end{aligned}
$$

where the subscript with respect to $\xi$ denotes derivative with respect to $\xi$. Note that

$$
1-\cos (4 \theta)=\frac{8 \xi^{2}\left(1+x_{R \xi}\right)^{2}}{\left(1+2 x_{R \xi}+x_{R \xi}^{2}+\xi^{2}\right)^{2}} .
$$

In Eq. (3), $P$ (Péclet number) is defined as

$$
P=\frac{U a}{2 D}
$$

where $U$ is the velocity of the advancing interface, $a$ the radius of curvature at the tip for the dendrite corresponding to the zero-surface-tension solution, $D$ the temperature diffusion constant. $d_{0}$ is the dimensional capillary parameter given by

$$
d_{0}=\frac{\gamma c T_{m}}{\tilde{l}^{2} a},
$$

where $\gamma$ is the surface tension, $c$ the specific heat per unit volume; $T_{m}$ is the melting temperature, $\widetilde{l}$ the latent heat. In this paper, it will be assumed that for any given $P, d_{0}$ is small enough so that $d_{0} / P<<1$. This is not a severe restriction since the theory presented here is valid for small $d_{0}$. The parameter $\Delta$ in (1) is the nondimensional undercooling defined as

$$
\Delta=\frac{c}{\widetilde{l}}\left(T_{m}-T_{\infty}\right)
$$

where $T_{\infty}$ denotes the temperature at $z=\infty$ far ahead of the finger. Note that each of $x$ and $z$ appearing in (3) are nondimensionalized by $a$. Also note that for nonzero $d_{0}$, the tip radius of curvature is not $a$ but $a\left[1+x_{R \xi}(0)\right]^{2}$ as can be seen from (6). Also, in the definition of $r$ in (4), the choice of a specific branch of the square root is made so that $r \geq 0$ for $\xi \geq \xi^{\prime}$ and $r<0$ otherwise for $\xi$ and $\xi^{\prime}$ on the real axis. Thus the absolute value $|r|$ appearing in (3) is needed to be in accordance with the Nash-Glicksman derivation. The choice of a specific branch in (4) is made for the purposes of analytic continuation of (3) to the complex $x$ plane as shall be seen later.

When surface tension is neglected, i.e., $d_{0}=0$, Ivantsov found exact solutions for a steadily growing dendrite with a parabolic interface shape with tip radius $a$ (which is arbitrary). In our notation and nondimensionalization, this corresponds to the exact solution

$$
z_{R}=0
$$

to (3). This is not immediately obvious on substitution of (11) into (3). However, Pelce and Pomeau have verified that $(11)$ is indeed the solution to (3) provided the undercooling $\Delta$ is related to the Péclet number $P$ by the relation

$$
\Delta=\pi^{1 / 2} P^{1 / 2} e^{P} \operatorname{erfc}\left(P^{1 / 2}\right) .
$$

Thus, when surface tension is neglected, it is clear from (8) and (12) that for given undercooling and other experimental conditions, only the product of dendrite velocity $U$ and the tip radius of curvature $a$ are determined. However, experimental evidence ${ }^{7,8}$ suggests that each of these two quantities are each separately determined as a function of the undercooling for other given experimental conditions. Thus, for an adequate theory, the degeneracy of these solutions needs to be removed. As pointed out earlier, any amount of surface tension introduces another parameter $d_{0}$ into the problem and therefore there is then enough dimensional information for unique determination of each of $U$ and $a$ separately. However, this does not guarantee that such a solution will exist and indeed our results suggest that in accordance with earlier numerical and analytical results (for restricted cases), solutions exist only when the crystalline anisotropy parameter $\alpha \neq 0$.

We now would like to simplify the integral expression on the right-hand side of (3). We will assume that for small $d_{0}$ and fixed $\xi, x_{R}$ is small. However, as shown in the Appendix, the boundary condition that the nondimensional temperature on the interface approach $\Delta$, a constant, as $\xi \rightarrow \pm \infty$ can allow for the interface shape correction function $x_{R}$ to grow with $\xi$ at a rate like $\xi^{1-s}$, where $s>0$. Thus $x_{R}$ need not uniformly be small. However, it is reasonable to assume that $x_{R \xi}$ is small uniformly for all $\xi$, and thus from expression (4) for $r$ on application of the mean-value theorem on the quantity 


$$
\frac{x_{R}(\xi)-x_{R}\left(\xi^{\prime}\right)}{\xi-\xi^{\prime}}
$$

it is clear that the deviation of $r$ from $r_{0}$ is small for small $d_{0}$ for all $\xi$ and $\xi^{\prime}$, where

$$
r_{0}=\left(\xi-\xi^{\prime}\right)\left[1+\frac{1}{4}\left(\xi+\xi^{\prime}\right)^{2}\right]^{1 / 2}
$$

Thus it is legitimate to linearize the right-hand side of (3) for any given $P$ for sufficiently small $d_{0}$. If we subtract off the Ivantsov solution, we find that to linear order in $x_{R}$ on the right-hand side:

$$
\begin{aligned}
-d_{0}\{1+\alpha[1-\cos (4 \theta)\} \kappa= & \frac{P}{\pi} \int_{-\infty}^{\infty} d \xi^{\prime} x_{R \xi^{(}}\left(\xi^{\prime}\right) e^{P\left[z\left(\xi^{\prime}\right)-z(\xi)\right]} K_{0}\left(P\left|r_{0}\right|\right) \\
& +\frac{P^{2}}{\pi} \int_{-\infty}^{\infty} d \xi^{\prime} e^{P\left[z\left(\xi^{\prime}\right)-z(\xi)\right.} \frac{K_{1}\left(P\left|r_{0}\right|\right)}{\left|r_{0}\right|}\left(\xi-\xi^{\prime}\right)\left[x_{R}\left(\xi^{\prime}\right)-x_{R}(\xi)\right] .
\end{aligned}
$$

Note that in (14), the subscript with respect to $\xi$ denotes derivative with respect to $\xi$. In (14), we used the identity that derivative of $K_{0}$ is $-K_{1}$. It is convenient to eliminate the $x_{R \xi}$ term by integrating by parts and we find after careful consideration of the singular nature of the integrand that (14) is equivalent to

$$
\begin{aligned}
-d_{0}\{1+\alpha[1-\cos (4 \theta)]\} \kappa= & x_{R}(\xi) \frac{P^{2}}{\pi} f_{-\infty}^{\infty} d \xi^{\prime} e^{P\left[z\left(\xi^{\prime}\right)-z(\xi)\right]} \frac{K_{1}\left(P\left|r_{0}\right|\right)}{\left|r_{0}\right|}\left(\xi^{\prime}-\xi\right) \\
& \left.-\frac{P^{2}}{\pi} f_{-\infty}^{\infty} d \xi^{\prime} e^{P\left[z\left(\xi^{\prime}\right)-z(\xi)\right]} x_{R}\left(\xi^{\prime}\right) z_{\xi}\left(\xi^{\prime}\right) \mid K_{0}\left(P\left|r_{0}\right|\right)+\frac{K_{1}\left(P\left|r_{0}\right|\right)}{\left|r_{0}\right|}\left[z(\xi)-z\left(\xi^{\prime}\right)\right]\right),
\end{aligned}
$$

where $f$ denotes principal value integral.

Now for the Ivantsov solution, the nondimensional temperature within the crystal is a constant, $\Delta$, and and so

$$
\Delta=\frac{P}{\pi} \int_{-\infty}^{\infty} d \xi^{\prime} e^{P\left[z\left(\xi^{\prime}\right)-z\right]} K_{0}\left(P\left|\widetilde{r}_{0}\right|\right)
$$

where $(x, z)$ is now inside the crystal and

$$
\widetilde{r}_{0}=\left[\left(x-\xi^{\prime}\right)^{2}+\left(z+\frac{1}{4} \xi^{\prime 2}\right)^{2}\right]^{1 / 2} .
$$

The partial derivative of the above expression with respect to $x$ must be zero, since the temperature within the crystal is uniform for the Ivantsov solution, when the curvature effects are neglected. On the other hand, if we take the derivative of the right-hand side of (16) with respect to $x$ and approach the interface from the inside of the crystal we find that

$$
-\frac{P^{2}}{\pi} f_{-\infty}^{\infty} e^{P\left[z\left(\xi^{\prime}\right)-z(\xi)\right]} \frac{K_{1}\left(P\left|r_{0}\right|\right)}{\left|r_{0}\right|}\left(\xi^{\prime}-\xi\right) d \xi^{\prime}+\frac{P z_{\xi}}{\left(1+z_{\xi}^{2}\right)}=0 \text {. }
$$

Thus (15) can be further simplified as

$$
\begin{aligned}
-d_{0}\{1+\alpha[1-\cos (4 \theta)]\} \kappa=\frac{P z_{\xi}}{\left(1+z_{\xi}^{2}\right)} x_{R}(\xi)-\frac{P^{2}}{\pi} f_{-\infty}^{\infty} e^{P\left[z\left(\xi^{\prime}\right)-z(\xi)\right]} x_{R}\left(\xi^{\prime}\right) z_{\xi}\left(\xi^{\prime}\right) \\
\quad \times\left\{K_{0}\left(P\left|r_{0}\right|\right)+\frac{K_{1}\left(P\left|r_{0}\right|\right)}{\left|r_{0}\right|}\left[z(\xi)-z\left(\xi^{\prime}\right)\right]\right] d \xi^{\prime} .
\end{aligned}
$$

\section{REGULAR PERTURBATION EXPANSION AND ANALYTICAL CONTINUATION TO THE UPPER HALF $\xi$ PLANE}

If we now carry out a regular perturbation expansion of $x_{R}$ in powers of $d_{0}$ :

$$
x_{R}(\xi)=d_{0} x_{1}(\xi)+d_{0}^{2} x_{2}(\xi)+\cdots
$$

we find that $x_{1}$ satisfies the linear singular integral equation

$$
\begin{aligned}
-\left\{1+\alpha\left[1-\cos \left(4 \theta_{0}\right)\right]\right\} \kappa_{0}=\frac{P z_{\xi}}{\left(1+z_{\xi}^{2}\right)} x_{1}(\xi)-\frac{P^{2}}{\pi} f_{-\infty}^{\infty} e^{P\left[z\left(\xi^{\prime}\right)-z(\xi)\right]} x_{1}\left(\xi^{\prime}\right) z_{\xi}\left(\xi^{\prime}\right) & \\
& \times\left[K_{0}\left(P\left|r_{0}\right|\right)+\frac{K_{1}\left(P\left|r_{0}\right|\right)}{\left|r_{0}\right|}\left[z(\xi)-z\left(\xi^{\prime}\right)\right]\right] d \xi^{\prime},
\end{aligned}
$$


where $\theta_{0}$ and $\kappa_{0}$ are equal to the expressions (5) and (6) for $\theta$ and $\kappa$ with the substitution $x_{R}=0$.

We numerically calculated a smooth solution to (20) by discretization and satisfying the equation at a discrete set of points. In addition to (20), we imposed the condition $x_{1}(0)=0$ so that the tip of the dendrite coincides with $(x, z)=(0,0)$. The resulting linear system was solved without any difficulties and consistency of the solution checked by doubling each of the number of discretization points and the size of the truncated domain. The solution, as expected, was found to be an odd function of $\xi$ implying a smooth symmetric dendrite at least to order $d_{0}$. In particular, this implies that the tip of the needle crystal is smooth. It is conceivable that the same is true to every order in the expansion (19) though we have not calculated higher-order solutions. We assume that this is indeed the case.

At this point, it is appropriate to point out that if instead of the parametric representation $(x(\xi), z(\xi))$, for the free boundary, we had used $(x, z(x))$ representation and decomposed

$$
z(x)=-\frac{1}{2} x^{2}+\widetilde{z}_{R}(x)
$$

and carried out a linearization of the integral term in the Nash-Glicksman equation, we would arrive precisely at (18) with $-z_{\xi} x_{R}(\xi)$ replaced by $\widetilde{z}_{R}(x)$, with $\xi$ and $\xi^{\prime}$ replaced by $x$ and $x^{\prime}$ and $z(\xi)$ replaced by $-\frac{1}{2} x^{2}$. However, justification of the linearization of the integral term of the Nash-Glicksman equation for such a representation appears to be difficult if such a representation were used.

Now, we proceed to calculate the leading-order transcendentally small correction to (19). Following the ideas of Kruskal and Segur, we do so by analytically continuing (18) to the upper half $\xi$ plane to find sources of nonuniformity of the expansion (19). These sources of nonuniformity in the complex $\xi$ plane contribute transcendentally small terms in the asymptotic expansion of $x_{R}$ and it is our intention to calculate the leading-order transcendentally small term in order to find any constraint on the parameter $d_{0}$ arising from the requirement that the tip of the parabola be smooth. This constraint is off course used to determine $d_{0}$ to the leading order. As higher-order transcendentally small terms are taken into account one has to correct the constraint on $d_{0}$ as well.
If we symbolically denote the actual constraint coming out of the requirement of a smooth tip to be $S\left(d_{0}\right)=$ a number, the determination of leading-order constraint is equivalent to replacing $S\left(d_{0}\right) \approx S^{\prime}(0) d_{0}$ in the above constraint. This is a valid approximation when $S^{\prime}(0)$ is nonzero and $d_{0}$ sufficiently small ( $S^{\prime}$ being assumed continuous). It is appropriate to point out that in most cases, transcendentally small terms that are beyond all orders of a regular perturbation expansion are mathematically meaningless because there is no distinction between two asymptotic expansions which are different from each other by a transcendentally small amount. However, in this case, we have the extraordinary situation that every term of the regular perturbation expansion for $x_{R}$ has a vanishing real part on an interval on the imaginary $\xi$ axis adjoining $\xi=0$; however, transcendentally small terms in $d_{0}$ do not generally have a vanishing real part on any interval adjoining $\xi=0$. Thus the real part of the transcendentally small term, where nonzero, dominates the regular perturbation terms on some on part of the imaginary $\xi$ axis and is therefore well defined. This point is discussed at length by Kruskal and Segur but is reiterated here since it appears to have been misunderstood. ${ }^{20}$ The condition that transcendentally small terms in $d_{0}$ also have vanishing real part on some segment of the imaginary $\xi$ axis adjoining $\xi=0$ follows from the requirement of a smooth tip and has to be enforced for a consistent solution. When this condition is violated for the leadingorder transcendentally small term, it will be seen as evidence that acceptable solutions do not exist as the smoothness condition has to be satisfied to every order of transcendental correction.

We now proceed with the determination of transcendentally small terms in $d_{0}$. It is convenient to define

$$
z_{R}(\xi)=\xi x_{R}(\xi)
$$

Note that $z_{R}(\xi)$ is not defined as $\widetilde{z}_{R}(x(\xi))$; however, when $\xi=O$ (1), to the leading order in $d_{0}$, the two are the same. Note that there can be deviation of $z_{R}(\xi)$ from $\widetilde{z}_{R}(x(\xi))$ which is not uniformly small for all $\xi$ even for small $d_{0}$.

If we restrict our attention to symmetric needle crystals for which $z_{R}(\xi)=z_{R}(-\xi)$ and substitute (22) into (18), then

$$
\begin{aligned}
& -d_{0}\{1+\alpha[1-\cos (4 \theta)]\} \kappa \\
& =-\frac{P}{\left(1+z_{\xi}^{2}\right)} z_{R}(\xi)+\frac{P^{2}}{\pi} f_{-\infty}^{0} e^{(P / 2)\left(\xi^{2}-\xi^{\prime 2}\right)} z_{R}(\xi)^{\prime}\left[K_{0}\left(P\left|r_{0}\right|\right)+K_{0}\left(P r_{1}\right)\right. \\
& \left.\quad+\frac{1}{2}\left[\frac{K_{1}\left(P\left|r_{0}\right|\right)}{\left|r_{0}\right|}+\frac{K_{1}\left(P r_{1}\right)}{r_{1}}\right]\left(\xi^{\prime 2}-\xi^{2}\right)\right] d \xi^{\prime},
\end{aligned}
$$

where

$$
r_{1}=-\left(\xi+\xi^{\prime}\right)\left[1+\frac{1}{4}\left(\xi-\xi^{\prime}\right)^{2}\right]^{1 / 2}
$$

We note that with the choice of branch in the above square root $r_{1} \geq 0$, for $\xi$ and $\xi$ on the negative real axis. Further

$$
r=|r|
$$


for $\xi>\xi^{\prime}$, and for $\xi<\xi^{\prime}$, we have to choose

$$
r=|r| e^{i \pi}
$$

in order to analytically continue to the upper half complex $\xi$ plane. It is well known that

$$
K_{0}\left(r e^{-i \pi}\right)=K_{0}(r)+i \pi I_{0}(r) .
$$

Thus (23) can be seen as the limit of $\xi$ approaching the negative real axis from the upper half complex $\xi$ plane of the following equation:

$$
-d_{0}\{1+\alpha[1-\cos (4 \theta)]\} \kappa(\theta)=l(\xi) z_{R}(\xi)+\int_{-\infty}^{0} d \xi^{\prime} G\left(\xi, \xi^{\prime}\right) z_{R}\left(\xi^{\prime}\right)+\int_{\xi}^{0} d \xi^{\prime} J\left(\xi, \xi^{\prime}\right) z_{R}\left(\xi^{\prime}\right)
$$

where

$$
\begin{aligned}
& G\left(\xi, \xi^{\prime}\right)=\frac{P^{2}}{\pi} e^{P\left(\xi^{2}-\xi^{\prime 2}\right) / 2}\left[K_{0}\left(\operatorname{Pr}_{0}\right)+K_{0}\left(\operatorname{Pr}_{10}\right)+\frac{1}{2}\left(\frac{K_{1}\left(\operatorname{Pr}_{0}\right)}{r_{0}}+\frac{K_{1}\left(\operatorname{Pr}_{1}\right)}{r_{1}}\right)\left(\xi^{\prime 2}-\xi^{2}\right)\right], \\
& J\left(\xi, \xi^{\prime}\right)=i P^{2} e^{P\left(\xi^{2}-\xi^{\prime 2}\right) / 2}\left[I_{0}\left(P r_{0}\right)+\frac{1}{2}\left(\xi^{2}-\xi^{\prime 2}\right) \frac{I_{1}\left(P_{0}\right)}{r_{0}}\right],
\end{aligned}
$$

and

$$
l(\xi)=-\frac{P}{1-i \xi} .
$$

From (20), in an analogous procedure, it is found that the analytical continuation of the leading-order regular perturbation solution $z_{1}(\xi) \equiv \xi x_{1}(\xi)$ in the upper half complex $\xi$ plane satisfies

$$
-\left\{1+\alpha\left[1-\cos \left(4 \theta_{0}\right)\right]\right\} \kappa_{0}=l(\xi) z_{1}(\xi)+\int_{-\infty}^{0} d \xi^{\prime} G\left(\xi, \xi^{\prime}\right) z_{1}\left(\xi^{\prime}\right)+\int_{\xi}^{0} d \xi^{\prime} J\left(\xi, \xi^{\prime}\right) z_{1}\left(\xi^{\prime}\right) .
$$

It is easy to see from (31) that $z_{1}(\xi)$ is singular at $\xi=i$ in the upper half complex $\xi$ plane. From symmetry of the equation, it is easy to see that $z_{1}$ is also singular at the lower half complex $\xi$ plane at $\xi=-i$. Thus we need to find local equations in the neighborhoods of these points such that as the real axis is approached, the solution matches with the regular perturbation expansion (19). The terms that will not match must be transcendentally small in the physical domain.

To find the form of the leading-order transcendentally small term, we subtract $d_{0}$ times (31) from (27) assuming that $z_{R}$ is a small deviation from $d_{0} z_{1}$ to find that the resulting homogeneous part of the equation for the small deviation $z_{H}$ is

$$
\begin{aligned}
z_{H}^{\prime \prime}\left(1+\frac{8 \alpha \xi^{2}}{\left(1+\xi^{2}\right)^{2}}\right)+z_{H}^{\prime}\left[-\frac{3 \xi}{\left(1+\xi^{2}\right)}-\frac{56 \alpha \xi^{3}}{\left(1+\xi^{2}\right)^{3}}+\frac{16 \alpha \xi}{\left(1+\xi^{2}\right)^{2}}\right) & +\frac{P}{d_{0}(1-i \xi)}\left(1+\xi^{2}\right)^{3 / 2} z_{H} \\
& =z_{H}\left[-\frac{3}{\left(1+\xi^{2}\right)}-\frac{56 \alpha \xi^{2}}{\left(1+\xi^{2}\right)^{3}}+\frac{16 \alpha}{\left(1+\xi^{2}\right)^{2}}\right]+I_{4}+I_{5}
\end{aligned}
$$

where

$$
I_{4}=-\frac{\left(1+\xi^{2}\right)^{3 / 2}}{d_{0}} \int_{-\infty}^{0} d \xi^{\prime} G\left(\xi, \xi^{\prime}\right) z_{H}\left(\xi^{\prime}\right)
$$

and

$$
I_{5}=-\frac{\left(1+\xi^{2}\right)^{3 / 2}}{d_{0}} \int_{\xi}^{0} d \xi^{\prime} J\left(\xi, \xi^{\prime}\right) z_{H}\left(\xi^{\prime}\right),
$$

\section{TRANSCENDENTALLY SMALL TERMS FOR $\alpha=0$}

For $\alpha=0$, i.e., no crystalline anisotropy, the leadingorder asymptotic solution for small $d_{0}$ to the linear integro-differential equation (32) in the upper complex $\xi$ plane away from the immediate neighborhood of the turning point $\xi=i$ must be linear combinations of $g_{1}$ and $g_{2}$ defined as

$$
\begin{aligned}
g_{1,2}= & \left(1+\xi^{2}\right)^{3 / 8}(1-i \xi)^{1 / 4} \\
& \times \exp \left[ \pm i P^{1 / 2} d_{0}^{-1 / 2} \int_{i}^{\xi} d \xi^{\prime}\left(1+\xi^{\prime 2}\right)^{3 / 4}\right. \\
& \left.\times\left(1-i \xi^{\prime}\right)^{-1 / 2}\right) .
\end{aligned}
$$

Note that the above is just the two independent WKB solutions to (32) with the right-hand side of (32) neglected. On substitution of (35) back into $I_{4}$ and $I_{5}$ it is clear that these contributions are of smaller order in $d_{0}$ compared to other terms on the left-hand side of (32). We note that on the imaginary $\xi$ axis in the interval $(0, i) g_{2}$ is real and transcendentally small, while $g_{1}$ is transcendentally large. This is also true for a certain region in the 
complex $\xi$ plane in the neighborhood of the imaginary axis in the interval $(0, i)$ (sector I as sketched in Fig. 1). However, there is another region in the complex $\xi$ plane with $\operatorname{Re} \xi<0$, where $g_{1}$ is transcendentally small and $g_{2}$ transcendentally large (sector II in Fig. 1). The boundary between these two sectors is called a Stokes line and is determined by the condition

$$
\operatorname{Re}\left[i \int_{i}^{\xi} d \xi^{\prime}\left(1+\xi^{\prime 2}\right)^{3 / 4}\left(1-i \xi^{\prime}\right)^{-1 / 2}\right]=0
$$

The sketch in Fig. 1 is justified from the following consideration: First we note that

$$
i \int_{i}^{0} d \xi^{\prime}\left(1+\xi^{\prime 2}\right)^{3 / 4}\left(1-i \xi^{\prime}\right)^{-1 / 2}
$$

is purely real and positive. Again,

$$
\operatorname{Re}\left[i \int_{0}^{\xi} d \xi^{\prime}\left(1+\xi^{\prime 2}\right)^{3 / 4}\left(1-i \xi^{\prime}\right)^{-1 / 2}\right]<0
$$

for $\xi$, on the negative real axis. By considering the argument of $\left(1-i \xi^{\prime}\right)^{-1 / 2}$, one easily establishes that the lefthand side of the above equation is a monotonically decreasing function of $|\xi|$ on the negative real axis. Using this, it is easy to show that only one of the Stokes lines emanating from the turning point $\xi=i$ intersects the negative real $\xi$ axis as shown in Fig. 1 .

Including the leading-order transcendentally small correction, in sector I (that includes the imaginary $\xi$ axis between 0 and $i$ ),

$$
z_{R} \sim d_{0} z_{1}+\odot+C_{1} g_{2},
$$

where $\mathcal{C}$ stands for higher-order corrections with power dependence in $d_{0}$. From now on, we do not bother to write $C$ though such terms are present in the expression for $z_{R}$ and dominate the leading-order transcendental correction in $d_{0}$ which will be explicitly written down as they determine the velocity selection.

As $\xi=0$ is approached, the importance of the transcendental term arising due to the effect of singularity at $\xi=-i$ becomes as important. At exactly $\xi=0$, the contribution of singularities at $\xi= \pm i$ are of the same order in $d_{0}$. Since $z_{R}$ must be real on the entire real $\xi$ axis, it follows that on the real $\xi$ axis in some neighborhood of $\xi=0$, the contribution from $\xi=-i$ to the leading order must be $C_{1}^{*} g_{2}^{*}$ (the asterisk denotes complex conjugate) so that on the real $\xi$ axis

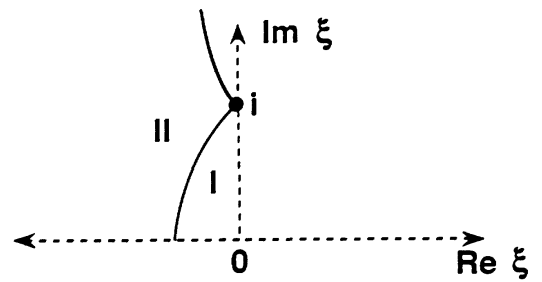

FIG. 1. Solid lines: Stokes lines determined by $\operatorname{Re}\left[i \int_{i}^{\xi} d \xi^{\prime}\left(1+\xi^{\prime 2}\right)^{3 / 4}\left(1-i \xi^{\prime}\right)^{-1 / 2}\right]=0$ in the complex $\xi$ plane for $\operatorname{Re} \xi \leq 0$.

$$
z_{R} \sim d_{0} z_{1}+C_{2} g_{2}+C_{2}^{*} g_{2}^{*}
$$

is real. It is easily seen that the slope at the tip as we approach it from the negative $\xi$ side is

$$
\frac{d z_{R}}{d \xi}=2 \operatorname{Re}\left[C_{1} g_{2}^{\prime}(0)\right] \text {. }
$$

On using (35)

$$
\begin{aligned}
\frac{d z_{R}(0)}{d \xi}= & 2 \operatorname{ImC} C_{1} P^{1 / 2} d_{0}^{-1 / 2} \\
& \times \exp \left[-i d_{0}^{-1 / 2} \int_{i}^{0} d \xi\left(1+\xi^{2}\right)^{3 / 4}\right. \\
& \left.\times(1-i \xi)^{-1 / 2}\right] .
\end{aligned}
$$

Thus a smooth tip implies

$$
\operatorname{Im} C_{1}=0 \text {. }
$$

In Sector II of Fig. 1, which borders on the real $\xi$ axis for sufficiently negative $\xi$, including the leading-order transcendental small correction, we must have

$$
z_{R} \sim d_{0} z_{1}+C_{2} g_{1}
$$

and in this sector, on the real axis we must have

$$
z_{R} \sim d_{0} z_{1}+C_{2} g_{1}+C_{2}^{*} g_{1}^{*} .
$$

To find $C_{1}$ and determine if the smooth tip condition (40) can be satisfied, we must consider the immediate neighborhood of $\xi=i$ in the upper half plane, where each of the expressions (37) and (41) are invalid both because of the linearization used in obtaining (32) and the fact that $\xi=i$ is a turning point. We introduce local dependent and independent variables $F$ and $\zeta$ defined as

$$
\begin{aligned}
& \xi=i\left(1-d_{0}^{2 / 7} P^{-2 / 7} 2^{-1 / 7} \zeta\right), \\
& z_{R}=-d_{0}^{4 / 7} P^{-4 / 7} 2^{-2 / 7} F .
\end{aligned}
$$

Then it is found that (35) is to the leading order in $d_{0}$ reduced to

$$
F^{\prime \prime}-\left(\zeta-F^{\prime}\right)^{3 / 2} F=1
$$

In obtaining (45) from (35), the contribution from $I_{4}$ is of order $d_{0}$ since $I_{4}$ involves the integral of $z_{R}$ on the real axis where $z_{R}=O\left(d_{0}\right)$. As far as $I_{5}$, one needs to be more careful, since in the range of integration includes the immediate neighborhood of $\xi=i$ where the scaling (44) holds. However, on careful analysis, it is found that $I_{5}$ does not contribute anything to the leading order as well. It is easily seen that the asymptotic behavior for large $\zeta$ that matches with $z_{R}=d_{0} z_{1}$ is

$$
F \sim-\frac{1}{\xi^{3 / 2}}
$$

To find transcendentally small correction to this, we linearize (45) about (46) and find that the homogeneous part of the linear equation is

$$
F_{H}^{\prime \prime}-\frac{3}{2 \zeta} F_{H}^{\prime}-\xi^{3 / 2} F_{H}=0
$$


The transcendental correction to (46) to the leading order for large $\zeta$ must be linear combinations of the WKB solutions to (47) given by

$$
\xi^{3 / 8} e^{ \pm(4 / 7) \xi^{7 / 4}}
$$

For $\operatorname{Arg} \xi$ in the interval $(-2 \pi / 7,0]$, inclusion of the leading-order transcendental correction gives

$$
F \sim-\frac{1}{\xi^{3 / 2}}+A_{1} \xi^{3 / 8} e^{-(4 / 7) \xi^{7 / 4}}
$$

This matches with (37) in sector I provided

$$
\frac{C_{1}}{A_{1}}=-2^{-6 / 7} P^{-13 / 28} d_{0}^{13 / 28}
$$

For large $\zeta$ with $\operatorname{Arg} \zeta$ in $\left(-\frac{6}{7} \pi, \frac{2}{7} \pi\right)$,

$$
F \sim-\frac{1}{\xi^{3 / 2}}+A_{2} \xi^{3 / 8} e^{(4 / 7) \xi^{7 / 4}}
$$

and this matches with (41) in sector II of Fig. 1 provided

$$
\frac{C_{2}}{A_{2}}=-2^{-6 / 7} P^{-13 / 28} d_{0}^{13 / 28}
$$

We note that we are interested in a solution to (45) which for large $\zeta$ with $\operatorname{Arg} \zeta$ in $(-6 \pi / 7,0]$ has the asymptotic behavior given by (46). Our numerical calculation of appropriate solution to (45) involved solving (45) on two rays emanating for $\xi=0$ going to a large distance from the origin with $\arg \xi=-\pi / 2$ and $\operatorname{Arg} \xi=0$ with asymptotic boundary condition (46) at the other end points of these straight-line contours. For a given trial value of $F(0)$ the two-point boundary problem on each ray was solved by standard second-order discretization of (45) and using Newton iteration. Once these solutions were obtained numerically, one-sided second-order differencing gave us the estimated value of $F^{\prime}(0)$ on each of the rays. In an outer Newton iterative procedure, the trial value of $F(0)$ was found so that the computed $F^{\prime}(0)$ along the two rays agree. From monitoring the size of the Jacobian, it was clear that the problem was not underdetermined and we checked that indeed a unique solution to (45) satisfying given decay conditions exist. Once the solutions converge, the imaginary part of solution $F$ along the ray coinciding with the positive real $\zeta$ axis at large distances was found to proportional to $\xi^{3 / 8} e^{-(4 / 7) \zeta^{7 / 4}}$ with the proportionality constant equaling -0.875 . From (49), it follows that $\operatorname{Im} A_{1}=-0.875$. From (42) and (50), the tip slope

$$
\frac{d z_{R}(0)}{d \xi}=-2^{1 / 7} P^{1 / 28} d_{0}^{-1 / 28} \operatorname{Im}\left(A_{1}\right) \exp \left(-i d_{0}^{-1 / 2} P^{1 / 2} \int_{i}^{0} d \xi^{\prime}\left(1+\xi^{\prime 2}\right)^{3 / 4}\left(1-i \xi^{\prime}\right)^{-1 / 2}\right]
$$

which on numerical evaluation is equal to

$$
2^{1 / 7} P^{1 / 28} d_{0}^{-1 / 28} 0.875 e^{-0.6156622 d_{0}^{-1 / 2} P^{1 / 2}},
$$

which is clearly nonzero. Going back to (49), it is easy to see that $\operatorname{Im} F$ is nonzero on the real $\zeta$ axis for large $\zeta$. This implies that $\operatorname{Re} x_{R}$ is nonzero on some segment of the imaginary $\xi$ axis adjoining $\xi=0$ implying that smooth tip solutions could not exist. The formal solution that we have constructed is an asymptotic solution of (1) for $\xi$ real in the interval $(-\infty, 0)$ where we relax the requirement of a smooth tip. This is not physically acceptable since a jump in the slope at the tip implies curvature which means that (1) could not possibly be satisfied at the tip. The same result with almost the same numerical values was obtained by Ben Amar and Pomeau for small Péclet number. Here, we see that (53) holds even for arbitrary Péclet number.

However, we differ with Ben Amar and Pomeau's analysis on a certain point which does not change the result (53) but is important as far as checking consistency of solution. They claim that the solution $F$ to (45) is singu- lar when $\xi \rightarrow 0$ and find the need of an inner neighborhood with a different scaling. Their argument is based on a possible behavior of (45) near the origin. However, not every solution to (45) need have a singular behavior at the origin and indeed from numerical integration of (45) (with careful choice of consistent branch cut), we find that the solution to (45) that satisfies the decay conditions at $\infty$ for $\operatorname{Arg} \zeta$ in $[0,6 \pi / 7)$ remains finite at $\zeta=0$. Indeed, if $F$ tends to $\infty$ as $\xi \rightarrow 0$, the linearization of the integral term in the Nash-Glicksman equation or even the PelcePomeau equation for complex $\xi$ in the neighborhood of $\xi=i$ would then be questionable.

However, this discrepancy with the analysis of Ben Amar and Pomeau has no bearing on the final results (53) which are in agreement.

\section{TRANSCENDENTALLY SMALL CORRECTION FOR NONZERO ANISOTROPY}

The WKB solutions to (32) for small $d_{0}$ are now given by $\widetilde{g}_{1}$ and $\widetilde{g}_{2}$, where

$$
\widetilde{g}_{1,2}=\left(1+\xi^{2}\right)^{7 / 4} L^{-1 / 4} \exp \left[\frac{1}{2} \int_{i}^{\xi} d \xi^{\prime}\left[Q\left(\xi^{\prime}\right)+\frac{7 \xi^{\prime}}{\left(1+\xi^{\prime 2}\right)}\right]\right] \exp \left[ \pm i d_{0}^{-1 / 2} \int_{i}^{\xi} d \xi^{\prime} L^{1 / 2}\left(\xi^{\prime}\right)\right]
$$


where

$L=\frac{P}{1-i \xi}\left(1+\xi^{2}\right)^{3 / 2}\left(1+\frac{8 \alpha \xi^{2}}{\left(1+\xi^{2}\right)^{2}}\right)^{-1}$,

$Q=\left(-\frac{3 \xi}{1+\xi^{2}}-\frac{56 \alpha \xi^{3}}{\left(1+\xi^{2}\right)^{3}}\right)\left[1+\frac{8 \alpha \xi^{2}}{\left(1+\xi^{2}\right)^{2}}\right)^{-1}$

Note that each of $L$ and $Q$ are singular at $\xi=\xi_{0}$ on the imaginary $\xi$ axis between 0 and $i$, where

$$
\xi_{0}=i\left[(1+2 \alpha)^{1 / 2}-(2 \alpha)^{1 / 2}\right] \text {. }
$$

The WKB solutions are invalid in a small neighborhood of $\xi=i$ and $\xi=\xi_{0}$. The form of the local equations depends on the size of $\alpha$. In the next two sections we consider two cases: $\alpha P^{4 / 7} d_{0}^{-4 / 7}=O(1)$ and $\alpha P^{4 / 7} d_{0}^{-4 / 7} \gg 1$.

\section{TRANSCENDENTAL CORRECTION FOR $\alpha P^{4 / 7} d_{0}^{-4 / 7}=O(1)$}

In this case $\xi_{0}$ is within a $d_{0}^{2 / 7}$ neighborhood of $i$. The WKB solutions (54) hold beyond a $d_{0}^{2 / 7}$ neighborhood of $\xi=i$ as in the preceding section. To the order of approximation to which $(54)$ is valid, we can replace $\widetilde{g}_{1}$ and $\widetilde{g}_{2}$ given by (54) by the simpler WKB solutions $g_{1}$ and $g_{2}$ as in (35). This is because in (55), $\alpha$ is small and the terms involving $\alpha$ are only important near $\xi=i$, where the WKB solutions are invalid anyway. Near $\xi=i$, we introduce the same change of variables (43) and (44) to find that the leading-order equation is now

$$
F^{\prime \prime}-\frac{\left(\zeta-F^{\prime}\right)^{7 / 2}}{\left[\left(\zeta-F^{\prime}\right)^{2}-\beta\right]} F=1
$$

where

$$
\beta=2^{9 / 7} \alpha P^{4 / 7} d_{0}^{-4 / 7} .
$$

As before, the asymptotic behavior of (58) that matches with $d_{0} z_{1}$ when $d_{0}^{2 / 7} \ll|1+i \xi|<<1$ is

$$
F \sim-\frac{1}{\zeta^{3 / 2}}
$$

We linearize (58) about this solution and obtain the transcendental correction to (60) from the WKB solutions of the form (48). Once again, as in the preceding section, (49) is valid for large $\zeta$ with $\operatorname{Arg} \zeta$ in $(-2 \pi / 7,0]$, and this matches with

$$
z_{R} \sim d_{0} z_{1}+C_{1} g_{2}
$$

in sector I (Fig. 1) provided (50) holds. Similarly, for large $\zeta$ with $\operatorname{Arg} \zeta$ in the interval $(-6 \pi / 7,-2 \pi / 7)$, (51) holds and this matches with (41) provided (52) holds. Thus a unique solution to $(58)$ is found by requiring that the solution goes to zero for large $\zeta$ with $\operatorname{Arg} \zeta$ in the interval $(-6 \pi / 7,0]$. However, for such a solution for arbitrary $\beta$, we generally have $\operatorname{Im} A_{1} \neq 0$ implying $\operatorname{Im} C_{1} \neq 0$. This implies a nonsmooth tip in the general case. However, on varying $\beta$, we obtain a set of values of $\beta$ and hence $d_{0}$ for a given $\alpha$ for which the smooth tip condition is satisfied. The smallest $\beta$ value was found numerically to be 1.4926. The details of the numerical method are given in Sec. VII. Note that the scaling of $d_{0}$ with $\alpha$ follows from the definition of $\beta$ and is consistent with earlier numerical $^{4,5}$ and analytical work. ${ }^{1,2}$ The results for the case of large $\beta$ with $\alpha<1$ are a special case of the case considered in the following section though it can be treated by a direct analysis of (58).

\section{TRANSCENDENTAL CORRECTION FOR $\alpha P^{4 / 7} d_{0}^{-4 / 7} \gg 1$}

Note that in this case, we could either have $\alpha=O$ (1) or $\alpha<1$ provided $\beta$ as defined by (59) is very much larger than unity. At the outset, we will be assuming that $\alpha$ is order unity. Later, scrutiny of the assumptions show that the final result is valid even for small $\alpha$ provided $\beta$ is large.

In this case, the WKB solutions (54) do not simplify to (35). The Stokes lines are determined by the condition

$$
\operatorname{Re}\left[i \int_{i}^{\xi} d \xi^{\prime} L^{1 / 2}\left(\xi^{\prime}\right)\right]=0
$$

where $L$ is given by (55). The Stokes lines in this case are shown in Fig. 2 and the asymptotic growths shown in sectors I and II are now relevant since they extend all the way to the negative real $\xi$ axis.

In this case, we introduce the independent and dependent variables in the neighborhood of $\xi=i$ given by

$$
\begin{aligned}
& \xi=i\left(1-d_{0}^{2 / 11} 2^{1 / 11} p^{-2 / 11} \alpha^{2 / 11} \xi\right) \\
& z_{R}=-d_{0}^{4 / 11} 2^{2 / 11} P^{-4 / 11} \alpha^{4 / 11} F
\end{aligned}
$$

Then the leading-order equation for $\zeta$ of $O(1)$ is

$$
F^{\prime \prime}+\left(\zeta-F^{\prime}\right)^{7 / 2} F=1
$$

For large $\zeta$, the asymptotic behavior that matches with $z_{R} \sim d_{0} z_{1}$ when $d_{0}^{2 / 7} \ll|1+i \xi| \ll<1$ is

$$
F \sim \frac{1}{\zeta^{7 / 2}}
$$

To find transcendentally small corrections to this behavior, we linearize (65) about this leading-order behavior

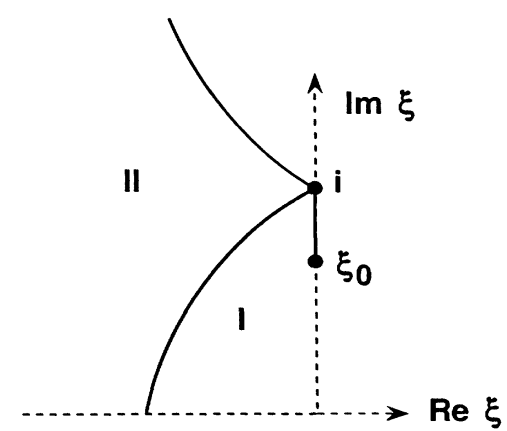

FIG. 2. Solid lines: Stokes lines determined by $\operatorname{Re}\left[i \int_{i}^{\xi} d \xi^{\prime} L^{1 / 2}\left(\xi^{\prime}\right)\right]=0$ in the complex $\xi$ plane for $\operatorname{Re} \xi \leq 0$, where $L$ is given by (55). 
and find WKB solutions to the homogeneous secondorder linear ordinary differential equation (ODE). Including this transcendental correction, we find that for large $\zeta$ with $\operatorname{Arg} \zeta$ in $(-4 \pi / 11,0)$

$$
F \sim \frac{1}{\zeta^{7 / 2}}+A_{1} \zeta^{7 / 8} e^{-i(4 / 11) \zeta^{11 / 4}}
$$

and this matches with

$$
z_{R} \sim d_{0} z_{1}+C_{1} \widetilde{g}_{2}
$$

in sector I of Fig. 2 provided

$$
\frac{C_{1}}{A_{1}}=-e^{i \pi / 4} P^{1 / 22} 2^{-39 / 22} \alpha^{-1 / 22} d_{0}^{9 / 44} .
$$

For large $\zeta$, for $\operatorname{Arg} \zeta$ in the interval $(-8 \pi / 11,-4 \pi / 11)$ the leading-order behavior of $F$ is given by

$$
F \sim \frac{1}{\zeta^{7 / 2}}+A_{2} \zeta^{7 / 8} e^{i(4 / 11) \xi^{11 / 4}}
$$

and this matches with

$$
z_{R} \sim d_{0} z_{1}+C_{2} \widetilde{g}_{1}
$$

in sector II (Fig. 2) provided

$$
\frac{C_{2}}{A_{2}}=-e^{i \pi / 4} P^{1 / 22} 2^{-39 / 22} \alpha^{-1 / 22} d_{0}^{9 / 44} .
$$

Thus a unique solution to (64) is calculated by requiring that the asymptotic behavior of the solution $F$ for large $\zeta$ be given by $(65)$ with only transcendentally small correction for $\operatorname{Arg} \xi$ in the entire interval $(-8 \pi / 11,0)$. It is clear that $A_{1}$ determined as such can only be a pure number. We do not determine this pure constant $A_{1}$ in this paper.

From the arguments similar to that of Sec. IV leading up to (40), it is clear that the appropriate condition for a smooth tip is that (67) be real on the imaginary $\xi$ axis near $\xi=0$. Thus it is necessary that

$\operatorname{Arg}\left[C_{1} \exp \left[-i d_{0}^{-1 / 2} \int_{i}^{\xi_{0}} d \xi^{\prime} L^{1 / 2}\left(\xi^{\prime}\right)\right]\right]=-n \pi$,

where $n$ is some positive integer. Note that the choice of a negative sign on the right-hand side of (72) follows from the sign of

$$
-d_{0}^{-1 / 2} \int_{i}^{\xi_{0}} d \xi^{\prime} L^{1 / 2}\left(\xi^{\prime}\right)
$$

which is negative because with the choice of branch, $\operatorname{Arg} L^{1 / 2}$ varies continuously from 0 to $\pi / 2 \operatorname{as} \operatorname{Arg}\left(\xi-\xi_{0}\right)$ varies from 0 to $-\pi$. Thus, from (68), we find that the condition of smooth tip implies that

$$
P^{1 / 2} d_{0}^{-1 / 2} G(\alpha)=n \pi+\frac{\pi}{4}+\operatorname{Arg} A_{1}
$$

where (see Table I)

$G(\alpha)=\int_{y_{0}}^{1} d y \frac{\left(1-y^{2}\right)^{3 / 4}}{(1+y)^{1 / 4}}\left[\frac{8 \alpha y^{2}}{\left(1-y^{2}\right)^{2}}-1\right]^{-1 / 2}$,

where
TABLE I. $G(\alpha)$ as defined in (74) for different $\alpha$.

\begin{tabular}{ll}
\hline \multicolumn{1}{c}{$\alpha$} & \multicolumn{1}{c}{$G(\alpha)$} \\
\hline 0.00001 & 0.0000756 \\
0.0001 & 0.000138 \\
0.001 & 0.00404 \\
0.01 & 0.0266 \\
0.02 & 0.0451 \\
0.03 & 0.0605 \\
0.04 & 0.0738 \\
0.05 & 0.0855 \\
0.06 & 0.0961 \\
0.07 & 0.106 \\
0.08 & 0.115 \\
0.09 & 0.123 \\
0.10 & 0.130 \\
0.12 & 0.144 \\
0.14 & 0.155 \\
0.16 & 0.166 \\
0.18 & 0.175 \\
0.20 & 0.183 \\
\hline \hline
\end{tabular}

$$
y_{0}=\xi_{0} / i=\sqrt{(1+2 \alpha)}-\sqrt{2 \alpha} .
$$

Equation (73) is the selection rule.

Equation (73) is also valid of small $\alpha$ only if $\beta$ is defined in (59) is very much larger than unity. In the case of small $\alpha$, it is easily seen that

$$
G(\alpha) \sim 2^{9 / 8} \alpha^{7 / 8} \int_{0}^{1} d q \frac{q^{7 / 4}}{\left(1-q^{2}\right)^{1 / 2}},
$$

which on numerical evaluation gives

$$
G(\alpha) \sim 1.80205 \alpha^{7 / 8} \text {. }
$$

In terms of $\beta,(73)$ reduces to

$$
\beta=1.2437\left[n \pi+\frac{\pi}{4}+\operatorname{Arg} A_{1}\right]^{8 / 7}
$$

Ben Amar and Pomeau also arrive at the result (73), but they implicitly assume that (73) is valid for any $\alpha$ and for any value of integer $n$. We claim that (73) can only strictly hold for large values of $n$ because if $n$ were of order unity, $\boldsymbol{G}(\alpha)$ in (73) will have to be small and of order $d_{0}^{1 / 2} P^{-1 / 2}$ (which has to be small for the theory to be valid). From (76), this would imply that $\beta=O(1)$ and then the result (73) is not strictly valid. In this case, one has to use the results of Sec. VI provided $\alpha$ is small. If $\alpha$ is not small, we cannot use any of the results of this paper or the previous ones ${ }^{1,2}$ to make a proper prediction of $d_{0}$ for the first few branches of solution, i.e., $n=O(1)$, since the corresponding $d_{0} P^{-1}$ are not small and therefore beyond the validity of the theory. However, despite the fact that (73) is strictly invalid for $n$ not large, it appears from comparison with direct numerical calculations ${ }^{1}$ that the formula is surprisingly accurate even for relative small $n$ and $\alpha$ over the range of experimental conditions.

Notice that the asymptotic form of solution (67) is also invalid near $\xi=\xi_{0}$ as pointed earlier by Ben Amar and Pomeau. However, this point has no bearing on the re- 
sult (73). If we are interested in finding the behavior of the solution in this neighborhood, we introduce local variables

$$
\xi=\xi_{0}-i t d_{0} \chi
$$

and

$$
z_{R}=-t^{2} d_{0}^{2} G(\chi),
$$

where

$$
t=\frac{-16 i \alpha \xi_{0}\left(1-\xi_{0}^{2}\right)\left(1-i \xi_{0}\right)}{P\left(1+\xi_{0}^{2}\right)^{9 / 2}} .
$$

Then to the leading order in $d_{0}$, the equation for $\chi=O(1)$ is

$$
\left(G^{\prime \prime}-1\right)\left(\chi-G^{\prime}\right)=G \text {. }
$$

For large $\chi, G \sim-\chi$ and linearizing (82) about this and finding WKB solutions to the associated homogeneous equation, we arrive at the following expression for $G$ for large $\chi$ that (with appropriate choice of constant $B$ ) matches with (67) as $\xi \rightarrow \xi_{0}$ for $\operatorname{Arg} \chi$ in $(-\pi, 0]$ :

$$
G \sim-\chi+B \chi^{-1 / 4} e^{-2 \chi^{1 / 2}} .
$$

This does not affect the selection rule (73).

\section{NUMERICAL DETERMINATION OF $\beta$ OF ORDER UNITY}

In this section, we describe the numerical method used to determine $\beta$ so that solution to Eq. (58) satisfies the asymptotic condition (60) for large $\zeta$ for $\operatorname{Arg} \xi$ in $(-6 \pi / 7,0]$ and that the solution be real on the positive real $\xi$ axis for $\zeta$ sufficiently large. From the Schwarz reflection principle, it follows that we are interested in a solution that satisfies the asymptotic decay condition (60) for large $\zeta$ when $\operatorname{Arg} \zeta$ in $(-6 \pi / 7,6 \pi / 7)$. Since only one such solution could be found, it follows that solution satisfying decay condition (60) for $\operatorname{Arg} \xi$ in $(-6 \pi / 7,6 \pi / 7)$ must automatically satisfy the condition that $F$ be real for sufficiently large $\xi$ on the real axis and indeed that was checked numerically.

The method employed is similar to the one employed earlier ${ }^{21}$ in the context of the Saffman-Taylor finger problem. We choose a point $x_{0}$ on the positive real axis that is sufficiently large so that the resulting solution is real at $\zeta=x_{0}$. This was done by trial and error. However, we do not choose $x_{0}$ unnecessarily large because such a choice will cause numerical inaccuracy.

We go through the procedure given in the next two paragraphs to calculate the residual corresponding to a given value of $\beta$.

We take $N$ points lined up parallel to the $\operatorname{Im} \zeta$ axis of the form $\xi_{k}=x_{0}-i L_{1}+i k h$, where $k$ is an integer ranging from 0 to $N+1, L_{1}$ is a large positive number far larger than $x_{0}$, and $h$ is the distance between adjacent $\xi_{k}$ points. $N$ is chosen to be an odd integer and $h$ chosen so that $(N+1) h=2 L_{1}$. The asymptotic condition (60) is employed at the end points $\zeta_{0}$ and $\zeta_{N+1}$ and the (58) discretized and satisfied at $\xi=\zeta_{k}$ for $k$ ranging from 1 to
$N$ using standard second-order finite differencing. This discretized two-point boundary-value problem is then solved using Newton iteration choosing an initial guess $F=0$ and convergence was obtained without any problems. Once convergence is attained, we store the value of $F$ and its estimated derivative obtained by second-order central differencing at $\zeta=x_{0}$.

The same procedure as in the last paragraph was used for a set of points on the real axis, $\zeta_{j}=x_{0}+j h_{1}$, where $j$ now ranges from 0 to $N_{1}+1$, with $\left(N_{1}+1\right) h_{1}=L_{2}$, where $L_{2}$ is a large positive number and $N_{1}$ is a large positive integer so that $h_{1}$ is small. Equation (58) is discretized and satisfied for $j=1, \ldots, N_{1}$ and the decay condition (60) used at end point corresponding to $j=N_{1}+1$. At the $j=0$ end point, we use the value of $F$ as obtained in the last paragraph. Once a converged solution is obtained on this contour, we estimate the derivative of $F$ at $\zeta=x_{0}$ by a one-sided second-order differencing. The real part of the difference of estimated derivative here and in the procedure of the last paragraph is the residual. The imaginary part is automatically zero to within machine precision, as it must be from the symmetry of the equation.

Once the residual is calculated for given $\beta$, in a Newton iterative procedure, we drive the residual to zero. The smallest value of $\beta$ so found was 1.4926 and this corresponds to the dendrite moving with the largest velocity. We took $N$ and $N_{1}$ to be 2049, $L_{1}$ and $L_{2}$ to be each 10 and $x_{0}=2.0$ and the results were unaffected by doubling each of $N, N_{1}$ or by changing $L_{1}, L_{2}$, and $x_{0}$. We do not carry out the calculation for other branches because experience has shown that (73) becomes quite accurate even for moderate values of $n$ though the expression should only be asymptotically valid for large $n$.

\section{DISCUSSION AND CONCLUSION}

We present here an analytic theory for the determination of velocity for a two-dimensional dendrite are arbitrary Péclet number in the limit of small values of the surface-tension parameter provided the ratio of surface tension and Péclet number is also small. We point out some discrepancies with earlier analytical work carried out in the limit of small Péclet number. The method is both qualitatively and quantitatively accurate and is an attempt to answer some serious objections raised by an earlier investigator on the validity of selection theory.

\section{ACKNOWLEDGMENTS}

This work was initiated following discussions with Martine Ben Amar who I wish to thank for introducing me to the dendrite problem. I am also grateful to Dan Meiron and Harvey Segur for fruitful discussions. A significant rewriting of an initial draft containing a less suitable free-boundary representation took place after discussion with Harvey Segur. This work was primarily supported by the National Science Foundation (Grant No. DMS-8713246). Partial funding was also provided by the National Aeronautics and Space Administration 
under Contract No. NAS1-18605 while the author was in residence at the Institute of Computer Applications in Science and Engineering.

\section{APPENDIX}

Define

$u[\xi] \equiv \frac{P}{\pi} \int_{-\infty}^{\infty} d \xi^{\prime}\left(1+\frac{d x_{R}}{d \xi}\right) e^{-P\left(\xi^{\prime 2}-\xi^{2}\right) / 2} K_{0}(P|r|)$

where

$$
\begin{aligned}
r= & \left\{\left(\xi-\xi^{\prime}\right)^{2}+2\left(\xi-\xi^{\prime}\right)\left[x_{R}(\xi)-x_{R}\left(\xi^{\prime}\right)\right]\right. \\
& \left.+\left[x_{R}(\xi)-x_{R}\left(\xi^{\prime}\right)\right]^{2}+\frac{1}{4}\left(\xi^{2}-\xi^{\prime 2}\right)^{2}\right\}^{1 / 2} .
\end{aligned}
$$

The purpose of this appendix is to show that the boundary condition

$$
\lim _{\xi \rightarrow \pm \infty} u(\xi)=\Delta
$$

can be satisfied for any shape correction function $x_{R}(\xi)$ satisfying the following bounds:

$$
\left|x_{R}(\xi)\right|<C_{1}\left(1+|\xi|^{1-s}\right)
$$

and

$$
\left|\frac{d x_{R}}{d \xi}\right|<\frac{C_{2}}{1+|\xi|^{s}}
$$

for some constants $C_{1}, C_{2}$, and $s$ with $C_{1}>0,1>C_{2}>0$, and $\frac{1}{2}>s>0$. Note that the $\Delta$ appearing on the righthand side of (A3) is related to $P$ through (12). Thus the shape correction from the Ivantsov parabola can actually grow at $\infty$. The upper bound on $C_{2}$ is not too restrictive since the function $d x_{R} / d \xi$ is expected to be small for $d_{0}$ reasonably small, though the proof does not assume anything directly about the size of $d_{0}$.

We will carry out the proof only for the symmetric dendrite, i.e., when $x_{R}(-\xi)=-x_{R}(\xi)$ though it is true in general. For a symmetric dendrite $(\mathbf{A} 1)$ reduces to

$$
\begin{gathered}
u[\xi]=\frac{P}{\pi} \int_{-\infty}^{0} d \xi^{\prime}\left(1+\frac{d x_{R}}{d \xi}\right) e^{-P\left(\xi^{\prime 2}-\xi^{2}\right) / 2} \\
\times\left[K_{0}(P|r|)+K_{0}\left(P r_{1}\right)\right]
\end{gathered}
$$

where

$$
\begin{aligned}
r_{1}=\{ & \left(\xi+\xi^{\prime}\right)^{2}+2\left(\xi+\xi^{\prime}\right)\left[x_{R}(\xi)+x_{R}\left(\xi^{\prime}\right)\right] \\
& \left.+\left[x_{R}(\xi)+x_{R}\left(\xi^{\prime}\right)\right]^{2}+\frac{1}{4}\left(\xi^{2}-\xi^{\prime 2}\right)^{2}\right\}^{1 / 2} .
\end{aligned}
$$

Choose any $s_{1}$ satisfying the condition

$$
1>s_{1}>1-s
$$

and choose

$$
\mu=(-\xi)^{s_{1}} .
$$

We assume $-\xi$ is large enough so that $-\xi+\mu<0$. We now decompose the function $u$

$$
u=u_{1}+u_{2}+u_{3}
$$

where

$$
\begin{aligned}
& u_{1}[\xi] \equiv \frac{P}{\pi} \int_{-\infty}^{\xi-\mu} d \xi^{\prime}\left(1+\frac{d x_{R}}{d \xi}\right) e^{-P\left(\xi^{\prime 2}-\xi^{2}\right) / 2}\left[K_{0}(P|r|)+K_{0}\left(\operatorname{Pr}_{1}\right)\right], \\
& u_{2}[\xi] \equiv \frac{P}{\pi} \int_{\xi-\mu}^{\xi+\mu} d \xi^{\prime}\left(1+\frac{d x_{R}}{d \xi}\right) e^{-P\left(\xi^{2}-\xi^{2}\right) / 2}\left[K_{0}(P|r|)+K_{0}\left(\operatorname{Pr}_{1}\right)\right], \\
& u_{3}[\xi] \equiv \frac{P}{\pi} \int_{\xi+\mu}^{0} d \xi^{\prime}\left(1+\frac{d x_{R}}{d \xi}\right) e^{-P\left(\xi^{\prime 2}-\xi^{2}\right) / 2}\left[K_{0}(P|r|)+K_{0}\left(\operatorname{Pr}_{1}\right)\right] .
\end{aligned}
$$

Now, from the properties of $K_{0}$ it is clear that there exists constant $B_{1}$ such that

$$
\begin{aligned}
& 0<P^{1 / 2}|r|^{1 / 2} K_{0}(P|r|) e^{P|r|}<B_{1}, \\
& 0<P^{1 / 2} r_{1}^{1 / 2} K_{0}\left(P r_{1}\right) e^{P r_{1}}<B_{1} .
\end{aligned}
$$

Thus

$$
\begin{aligned}
\left|u_{1}\right| & <\left(1-C_{2}\right) \frac{P^{1 / 2}}{\pi} B_{1} 2 \sqrt{2} \int_{-\infty}^{\xi-\mu} d \xi^{\prime} \frac{e^{-P\left(\xi^{\prime 2}-\xi^{2}\right)}}{\left(\xi^{\prime 2}-\xi^{2}\right)^{1 / 2}} \\
& <\left(1-C_{2}\right) P^{1 / 2} B_{1} 2 \frac{e^{2 P \xi}}{\sqrt{-\xi}} \int_{\mu}^{\infty} d q \frac{e^{-P q}}{\sqrt{q}}
\end{aligned}
$$

It is clear that in the limit of $\mu \rightarrow-\infty$, the right-hand side of (A16) goes to zero.

Now consider $u_{2}$ for large negative $\xi$. We have 


$$
\begin{aligned}
\left|u_{2}\right| & <\left(1-C_{2}\right) \frac{P^{1 / 2}}{\pi} B_{1} 2 \sqrt{2} \int_{\xi-\mu}^{\xi+\mu} d \xi^{\prime} \frac{e^{-P\left(\xi^{\prime 2}-\xi^{2}\right) / 2} e^{-P\left|\xi^{\prime 2}-\xi^{2}\right| / 2}}{\left(\left|\xi^{\prime 2}-\xi^{2}\right|\right)^{1 / 2}} \\
& <\frac{P^{1 / 2}}{\pi}\left(1-C_{2}\right) B_{1} 2 \sqrt{2} \int_{1+\mu / \xi}^{1-\mu / \xi} \frac{d q}{\left(\left|1-q^{2}\right|\right)^{1 / 2}} .
\end{aligned}
$$

It is clear that the right-hand side of (A17) goes to zero as $\xi \rightarrow-\infty$ and thus $u_{2}$ approaches zero as $\xi \rightarrow-\infty$.

It is now appropriate to break up $u_{3}(\xi)$ into two integrals:

$$
u_{3}(\xi)=u_{31}(\xi)+u_{32}(\xi)
$$

where

$$
\begin{aligned}
& u_{31}[\xi] \equiv \frac{P}{\pi} \int_{\xi+\mu}^{0} d \xi^{\prime} e^{-P\left(\xi^{\prime 2}-\xi^{2}\right) / 2}\left[K_{0}(P|r|)+K_{0}\left(\operatorname{Pr}_{1}\right)\right] \\
& u_{32}[\xi] \equiv \frac{P}{\pi} \int_{\xi+\mu}^{0} d \xi^{\prime} \frac{d x_{R}}{d \xi}\left(\xi^{\prime}\right) e^{-P\left(\xi^{\prime 2}-\xi^{2}\right) / 2}\left[K_{0}(P|r|)+K_{0}\left(\operatorname{Pr}_{1}\right)\right]
\end{aligned}
$$

Now

$$
\begin{aligned}
\left|u_{32}\right| & <\frac{P^{1 / 2}}{\pi} 2 \sqrt{2} B_{1} \int_{\xi+\mu}^{0} d \xi^{\prime}\left|\frac{d x_{R}}{d \xi^{\prime}}\right| \frac{1}{\left(\xi^{2}-\xi^{\prime 2}\right)^{1 / 2}} \\
& <\frac{P^{1 / 2}}{\pi} 2 \sqrt{2} B_{1} C_{2} \int_{\xi+\mu}^{0} d \xi^{\prime}\left(-\xi^{\prime}\right)^{-s} \frac{1}{\left(\xi^{2}-\xi^{\prime 2}\right)^{1 / 2}} \\
& <\frac{P^{1 / 2}}{\pi} 2 \sqrt{2} B_{1} C_{2}(-\xi)^{-s} \int_{0}^{1+\xi / \mu} d q q^{-s} \frac{1}{\left(1-q^{2}\right)^{1 / 2}} .
\end{aligned}
$$

It is easy to see that the right-hand side of (A21) goes to zero as $\xi \rightarrow-\infty$. Thus in this limit $u_{32} \rightarrow 0$.

We now consider $u_{31}$. First we have known asymptotic property of the modified Bessel function $K_{0}$; it is clear that for large enough argument, say larger than 10 , one can choose constant $C_{4}$, a pure number, so that

$$
\begin{aligned}
& \left|K_{0}(P|r|)-\frac{e^{-P|r|}}{\sqrt{P|r|}}\left(\frac{2}{\pi}\right)^{1 / 2}\right|<\frac{C_{3}}{P^{3 / 2}} \frac{e^{-P|r|}}{|r|^{3 / 2}}, \\
& \left|K_{0}\left(P_{1}\right)-\frac{e^{-P r_{1}}}{\sqrt{P r_{1}}}\left(\frac{2}{\pi}\right)^{1 / 2}\right|<\frac{C_{3}}{P^{3 / 2}} \frac{e^{-P r_{1}}}{r_{1}^{3 / 2}} .
\end{aligned}
$$

It is convenient to break up $u_{31}$ into two more integrals:

$$
u_{31}=u_{311}+u_{312} \text {, }
$$

where

$$
u_{311}=\left(\frac{2}{\pi}\right)^{1 / 2} \frac{P^{1 / 2}}{\pi} \int_{\xi+\mu}^{0} d \xi^{\prime} e^{-P\left(\xi^{\prime 2}-\xi^{\prime}\right) / 2}\left(\frac{e^{-P\left|r_{0}\right|}}{\sqrt{\left|r_{0}\right|}}+\frac{e^{-P r_{10}}}{\sqrt{r_{10}}}\right)
$$

and

$$
u_{312}=\int_{\xi+\mu}^{0} d \xi^{\prime} e^{-P\left(\xi^{\prime 2}-\xi^{2}\right) / 2}\left(\frac{e^{-P|r|}}{\sqrt{P\left|r_{0}\right|}} E\left(\xi, \xi^{\prime}\right)+\frac{e^{-P r_{10}}}{\sqrt{P r_{10}}} E_{1}\left(\xi, \xi^{\prime}\right)\right)
$$

where

$$
E\left(\xi, \xi^{\prime}\right)=\frac{e^{-P|r|+P\left|r_{0}\right|} \sqrt{\left|r_{0}\right|}}{\sqrt{|r|}}-1
$$

and

$$
E_{1}\left(\xi, \xi^{\prime}\right)=\frac{e^{-P\left|r_{1}\right|+P\left|r_{10}\right|} \sqrt{\left|r_{10}\right|}}{\sqrt{\left|r_{1}\right|}}-1
$$

In view of (A4) and (A5), it is easy to show that in the range of integration each of $E\left(\xi, \xi^{\prime}\right)$ and $E_{1}\left(\xi, \xi^{\prime}\right)$ are bounded above in absolute value by $B_{2} \mu^{-\left(s+s_{1}-1\right)}$, for some constant $B_{2}$ and so the latter term in (A26) is less than

$B_{2} P^{-1 / 2} \mu^{-\left(s+s_{1}-1\right)} \int_{\xi+\mu}^{0} d \xi^{\prime}\left(\xi^{2}-\xi^{\prime 2}\right)^{-1 / 2}$.

On substitution of $\xi^{\prime}=q \xi$ into the integral in (A29), it is easily seen that the contribution from (A29) tends to 0 as $\xi \rightarrow-\infty$. Thus in the limit of $\xi \rightarrow-\infty$, we are left only with the contribution from the first term in (A24) which is independent of $x_{R}$ and hence must be that from the Ivantsov solution. But it is known that the $u$ is equal to $\Delta$ for the Ivantsov solution. Thus the limit of the first term in (A26) in the limit of $\xi \rightarrow-\infty$ must be $\Delta$. Thus the proof is complete. 
${ }^{1}$ M. Ben Amar and Y. Pomeau, Europhys. Lett., 2, (4) 307 (1986).

${ }^{2}$ A. Barbeiri, D. C. Hong, and J. Langer, Phys. Rev. A 35, 1802 (1986).

${ }^{3}$ B. Caroli, C. Caroli, B. Roulet and J. Langer, Phys. Rev. A 22, 442 (1986).

${ }^{4}$ D. A. Kessler and H. Levine, Phys. Rev. A 33, 7867 (1986).

${ }^{5}$ D. I. Meiron, Phys. Rev. 33, 2704 (1986).

${ }^{6}$ G. P. Ivantsov, Dokl. Akad. Nauk. SSSR 58, 567 (1947).

${ }^{7}$ S.-C. Huang and M. E. Glicksman, Acta Metall. 29, 701 (1981); 29, 717 (1981).

${ }^{8}$ H. Honjo and Y. Sawada, J. Cryst. Growth 58, 297 (1982).

${ }^{9}$ J. S. Langer, Phys. Rev. A 33, 435 (1986).

${ }^{10}$ D. A. Kessler, J. Koplik, and H. Levine, Phys. Rev. A 31, 1712 (1985).
${ }^{11}$ M. Kruskal and H. Segur (unpublished).

${ }^{12}$ V. L. Pokrovskii and I. M. Khalatnikov, Zh. Eksp. Teor. Fiz. 40, 1713 (1961) [Sov. Phys. _JETP 13, 1207 (1961)].

${ }^{13}$ P. Pelce and Y. Pomeau, Stud. Appl. Math. 74, 245 (1986).

${ }^{14}$ G. E. Nash, Naval Research Laboratory, Report Nos. 7670 and 7680, 1978 (unpublished).

${ }^{15} \mathrm{R}$. Combescot, T. Dombre, V. Hakim, Y. Pomeau, and A. Pumir, Phys. Rev. Lett. 56, 2036 (1986).

${ }^{16}$ B. I. Shraiman, Phys. Rev. Lett. 56, 2028 (1986).

${ }^{17}$ J. J. Xu, Stud. Appl. Math. (to be published).

18 J. J. Xu (unpublished).

${ }^{19}$ A. Barbeiri and J. S. Langer (unpublished).

${ }^{20}$ J. J. Xu, McGill University report (unpublished).

${ }^{21}$ S. Tanveer, Phys. Fluids 30, 1589 (1987). 